\title{
ЯДЕРНО-ФІЗИЧНІ ДОСЛІДЖЕННЯ
}

\author{
УКРАЇНСЬКИЙ АНТАРКТИЧНИЙ \\ ЖУРНАЛ \\ УАЖ, № 10-11, 114-120, (2011/2012)
}

УДК 550.388.2

\section{ОПРЕДЕЛЕНИЕ КРИТИЧЕСКОЙ ЧАСТОТЫ Ғ-СЛОЯ ПО ДАННЫМ НАБЛЮДЕНИЙ ИОНОСФЕРНОГО АЛЬФВЕНОВСКОГО РЕЗОНАНСА}

\author{
А.В. Колосков, Н.А. Бару \\ Радиоастрономический институт НАН Украиньл; г. Xарьков; koloskov@ rian.kharkov.ua
}

Реферат. Параметры ионосферного альфвеновского (ИАР) и шумановского (ШР) резонансов определяются как интенсивностью их источника (мировой грозовой активности), так и свойствами среды распространения сигналов (ионосферы и магнитосферы Земли). Наши наблюдения и результаты других авторов показывают, что в то время как ШР зависят в первую очередь от глобальных параметров источника и среды распространения, свойства ИАР в основном определяются локальными характеристиками околоземной плазмы. Последнее обстоятельство дало основание авторам работы проанализировать возможность диагностики локальной ионосферы по данным мониторинга ИАР. Для анализа использованы СНЧ записи, полученные на Украинской антарктической станции (УАС) и Низкочастотной обсерватории РИНАН Украины (НЧО). Наличие многолетних массивов данных, полученных в точках с пространственным разносом порядка 15 тысяч километров, позволило изучить основные сезонные и суточные закономерности поведения собственных частот и “наблюдаемости” ИАР, а также исследовать зависимость этих параметров от положения приемных пунктов. Характеристики ИАР сопоставлялись с синхронными данными ионосферного зондирования, полученными на УАС, и величинами полного электронного содержания (ПЭС) над пунктами наблюдений. Совместный анализ данных показал, что определяющее влияние на вариации собственных частот максимумов ИАР оказывают параметры плазмы на высоте F-области ионосферы. В работе описана феноменологическая модель и предложена методика оценки критической частоты $\mathrm{F}$ слоя - $f_{0} F 2$ по величине частотного интервала между максимумами ИАР - $\Delta f$. Достоверность методики подтверждена сопоставлением восстановленных значений $f_{0} F 2$ с данными вертикального зондирования на УАС.

Ключевые слова: резонанс, Альфвен, критическая частота.

Визначення критичної частоти f-шару за даннми спостережень іоносферного альфвенівського резонансу. А.В. Колосков, Н.А. Бару

Реферат. Параметри іоносферного альфвенівського (IAP) та шуманівського (ШР) резонансів визначаються як інтенсивністю їх джерела (світова грозова активність), так і властивостями середовища поширення сигналів (іоносфери і магнітосфери Землі). Наші спостереження та результати інших авторів показують, що в той час як ШР залежать в першу чергу від глобальних параметрів джерела i середовища поширення, властивості IAP в основному визначаються локальними характеристиками навколоземної плазми. Остання обставина дала підставу авторам роботи проаналізувати можливість діагностики локальної іоносфери за даними моніторингу IAР. Для аналізу використані СНЧ записи, отримані на Українській антарктичній станції (УАС) і Низькочастотній обсерваторії РІНАН України (НЧО). Наявність багаторічних масивів даних, отриманих в точках 3 просторовим розносом близько 15 тисяч кілометрів, дозволила вивчити основні сезонні і добові закономірності поведінки власних частот і “спостережуваності” IAP, а також дослідити залежність цих параметрів від положення приймальних пунктів. Характеристики IAP зіставлялися з синхронними даними іоносферного зондування, отриманими на УАС, і величинами повного електронного змісту (ПЕЗ) над пунктами спостережень. Спільний аналіз даних показав, що визначальний вплив на варіації власних частот максимумів IAP надають параметри плазми на висоті F-області іоносфери. У роботі описано феноменологічну модель і запропоновано методику оцінки критичної частоти $\mathrm{F}$ шару - $f_{0} F 2$ 


\section{А.В. КоЛОсков: ОПРЕДЕЛЕНИЕ КРИТИЧЕСКОЙ ЧАСТОТЫ Ғ-СЛОЯ ПО ДАННЫМ ...}

за величиною частотного інтервалу між максимумами IAP - $\Delta f$. Достовірність методики підтверджено зіставленням відновлених значень $f_{0} F 2$ з даними вертикального зондування на УАС.

Ключові слова: резонанс, Альфвен, критична частота.

F-layer critical frequency determination from ionospheric Alfven resonance observations. A.V. Koloskov, N.A. Baru

Abstract. The parameters of the ionospheric Alfven (IAR) and Schumann (SR) resonances are determined by the intensity of their source (global thunderstorm activity) and properties of the propagation medium (the ionosphere and magnetosphere of the Earth). Our observations and results of other authors show that the SR depends primarily on the global parameters of the source and propagation medium. Otherwise the IAR features are mainly determined by local characteristics of the near-earth plasma. The latter circumstance was the basis for the authors to analyze the possibility of diagnostics of the local ionosphere using the IAR data. ELF recordings, obtained at the Ukrainian Antarctic Station (UAS) and low-frequency observatory IRA NAS Ukraine (LFO) were used for the analysis. The presence of long-term data sets obtained at the points with a spatial spacing of about 15 thousand kilometers, allowed us to study the basic seasonal and diurnal behavior of the eigenfrequencies and "observability" of IAR, as well as to investigate the dependence of these parameters from the site position. IAR characteristics were compared with synchronous data of the ionospheric sounding and the total electron content (TEC) over the observation points. A joint analysis of data showed that plasma parameters at the height of the ionosphere F-region have a major impact on the variation of eigenfrequencies of IAR. The article describes a phenomenological model and proposes the technique of estimation of the magnitude of the critical frequency of $F$ layer - $f_{0} F 2$ based on the calculation of frequency interval between the IAR peaks $-\Delta f$. The reliability of this technique is confirmed comparing the reconstructed values $f_{0} F 2$ with the vertical sounding data obtained at UAS.

Key words: resonance, ionosphere, Alfven, critical frequency

\section{1. Введение}

Ионосферный альфвеновский резонатор (ИАР) представляет собой резонансную систему для магнитогидродинамических волн, локализованную на высотах $\mathrm{F}$ области ионосферы. Верхняя граница ИАР соответствует диапазону высот с большим градиентом спадания альфвеновской скорости, связанным с областью быстрого уменьшения плотности плазмы выше максимума слоя F. На нижней границе резонансной полости (высоты Еобласти ионосферы) альфвеновские волны трансформируются в электромагнитные и формируют резонансные структуры спектра (РСС) в УНЧ диапазоне. Первые сведения об ИАР в литературе появились в 1981 г. (Поляков и др., 1981), когда теоретически была показана возможность образования в ионосфере резонансной системы для альфвеновских волн и была выполнена оценка верхней границы частотного диапазона РСС - 1 Гц. Впоследствии эта оценка была повышена до 10 Гц (Беляев и др., 1989) и подтверждена (Беляев и др., 1989б) экспериментально. Отметим, что до настоящего момента наблюдения РСС были ограничены полосой частот 0.1-10 Гц. Исследования ИАР проводились разными авторами, как в низких (Bosinger, 2002), так и в высоких широтах (Belyaev et al., 1999), рассматривались собственные частоты резонатора, вероятность регистрации, сезонные и суточные зависимости, связь с магнитной активностью и солнечным циклом (Belyaev et al., 2000), осуществлялись многопозиционные измерения (Parent et al., 2005). Однако большинство имеющихся в литературе работ обсуждают результаты единичных записей PCC, а сами измерения носят фрагментарный характер.

ИАР является ионосферным образованием, чутко реагирующим на изменение условий в ближнем космосе. Характеристики резонанса меняются вследствие регулярных и спорадических вариаций геофизических и гелиофизических факторов, составляющих космической погоды. В частности, проявляется эффект воздействий, мощных возмущений магнитного поля (бури и суббури), потоков высокоэнергетичных частиц, всплесков рентгеновского и ультрафиолетового излучения, сопровождающих вспышечную деятельность 
на Солнце, и, с другой стороны, солнечных затмений, ежедневных и сезонных вариаций освещенности на высотах ионообразования. Таким образом, мониторинг ИАР может быть эффективным способом слежения за космической погодой. Особенный интерес представляют синхронные измерения ИАР, осуществляемые в пунктах с большим пространственным разрешением, поскольку они позволяют селектировать отклик резонансов на локальные и глобальные процессы в геокосмосе. Данное исследование посвящено анализу результатов непрерывного двухпозиционного патруля ИАР, осуществляемого на Украинской антарктической станции и на низкочастотной обсерватории (НЧО, Украина). Работа обобщает результаты однопунктовых исследований ИАР (Колосков и др., 2008) на случай многопозиционных наблюдений. В ней обсуждаются особенности поведения ИАР в высоких (Антарктика) и средних (Украина) широтах, их связь с параметрами околоземной плазмы критической частотой ионосферы и полным электронным содержанием (ПЭС). Показана определяющая роль локальной ионосферы на формирование РСС. В работе впервые обнаружены случаи регистрации РСС на частотах до 30 Гц, что существенно превышает частотную границу наблюдений, известную из литературы. Также описана методика оценки величины критической частоты ионосферы по данным анализа собственных частот ИАР.

\section{2. Методика эксперимента и морфологические особенности поведения ИАР}

Мониторинг ИАР выполнялся в двух разнесённых пунктах - на высокоширотной

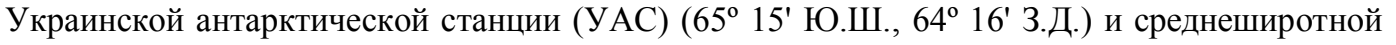
низкочастотной обсерватории РИ НАН Украины (НЧО) (49 56' С.Ш., $36^{\circ}$ 57' В.Д.). Станции находятся в близких L-оболочках, $\mathrm{L}=2,1$ на УАC и $\mathrm{L}=2.4$ на НЧО. Для исследования на НЧО использовался приемно-измерительный СНЧ комплекс, разработанный в РИ НАН Украины и предназначенный для мониторинга электромагнитного поля Земли в диапазоне 0.5-40 Гц. На УАС находится индукционный магнитометр Lemi112, разработанный Львовским центром Института космических исследований НАНУ НКАУ, с частотным диапазоном 0.01-300 Гц. Оба прибора позволяют проводить измерения вариаций ортогональных горизонтальных компонент магнитного поля в абсолютных единицах. Приёмные каналы разных поляризаций обладают высокой степенью идентичности. Более подробно с характеристиками приборов можно ознакомиться на сайтах изготовителей: http://ri.kharkov.ua/geospace/en/observatory/elf.html и http://isr.lviv.ua.

В работе проанализирован массив совместных данных за годовой интервал с ноября 2007 по ноябрь 2008 года. При первичной обработке данные усреднялись по 10 минутам. Далее строились суточные спектрограммы, для которых оператор обозначал спектральные максимумы, отвечающие ИАР. Таким образом, для каждого 10-минутного интервала восстанавливались факт присутствия дискретных линий в спектре и средний частотный разнос между ними. Кроме того, рассчитывалась вероятность регистрации (т.е. относительная частота появления резонансных максимумов в спектре), которая является более информативным параметром, чем сам факт регистрации РСС. Наличие длительных массивов данных позволило изучить сезонные и суточные зависимости основных характеристик ИАР. На УАС максимум вероятности регистрации приходится на 5 UT, а на НЧО на 21 UT, что соответствует локальной полночи в каждом из пунктов. В дневные часы на НЧО практически не видна резонансная структура, тогда как на УАС она проявляется, но с меньшей вероятностью. Также отмечается чётко выраженная зависимость вероятности регистрации по сезонам. Локальной зимой она очень высока и практически не зависит от времени суток. Весной резко падает и в полуденные часы ИАР не наблюдаются, летом резонансная структура видна только иногда в ночные часы. Осенью же вероятность регистрации достаточно высока, но есть отчетливая зависимость между ночными и дневными часами. Другой важной количественной характеристикой резонанса является разница между спектральными максимумами $d F$, которая определялась как среднее 


\section{А.В. КоЛОсков: ОПРЕДЕЛЕНИЕ КРИТИЧЕСКОЙ ЧАСТОТЫ Ғ-СЛОЯ ПО ДАННЫМ ...}

расстояние между каждой соседней парой максимумов резонансной структуры в данный момент времени. Среднесуточное распределение параметра (усредненное за год) характеризуется плавным ростом с вечера к утру и значительным уменьшением $d F$ в районе местного полдня. Также имеет место сезонная зависимость. Локальной зимой $d F$ наибольшая, затем происходит уменьшение к лету и быстрое увеличение осенью. Напомним, что УАС и НЧО находят в близких L-оболочках, и, как следствие, величины $d F$ на них сопоставимы.

Так как ИАР определяется состоянием ионосферы, то целесообразно найти связь между характеристиками резонатора и параметрами околоземной плазмы. Для этого вероятность регистрации РСС была сопоставлена с ПЭС и критической частотой слоя F2 ионосферы. На рис. 1 (рис.1-3, 5 см. на цв. вклейке между 112 и 113 стр.) представлены графики среднемесячной вероятности регистрации ИАР за год. Кривая соответствует величине ПЭС. Наблюдается обратная зависимость между уровнем электронной концентрации и вероятностью регистрации ИАР: чем ниже ПЭС, тем выше вероятность регистрации. Также обратим внимание на значительно более выраженный характер хода вероятности и большую величину его изменений в Антарктике по сравнению с Украиной. Это объясняется большей плотностью и меньшей вариабельностью ионосферы в средних широтах по сравнению с высокими. Аналогичная зависимость наблюдается и при сравнении характеристик ИАР с критической частотой слоя F2.

В отличие от выводов теоретических (Беляев и др., 1989а) и экспериментальных (Беляев и др., 1989б) исследований ИАР, оценивающих верхнюю границу диапазона наблюдения линий ИАР в 10 Гц, нами РСС регистрировались на значительно более высоких частотах. На рис. 2 представлена спектрограмма, полученная на НЧО для 6.12.2007, когда РСС наблюдалась в диапазоне частот до 30 Гц и выше.

За время анализа подобных случаев было зафиксировано более полусотни. Стоит отметить, что одновременно в обоих пунктах РСС на высоких частотах практически не регистрировались. За рассматриваемый период такое произошло только дважды. В остальные дни наблюдений РСС выше 10 Гц фиксировалась только на одной из станций. Максимум регистрации явления относится к интервалам локальной зимы, ранней весны и поздней осени, т.е. ко времени наибольшей вероятности регистрации ИАР, хотя зафиксированы единичные случаи и в другие сезоны года. Данный факт подтверждает предположение о том, что характеристики ИАР зависят в большей мере не от глобальных ионосферных условий, а от параметров околоземной плазмы над пунктом наблюдения. Период с ноября 2007 по ноябрь 2008 года не отличался значительной солнечной активностью, локальный К-индекс редко превышал фактор 3. Поэтому не было возможности исследовать поведение ИАР при высоких уровнях магнитной активности. Однако для значений К-индекса от 0 до 3 была отмечена четко выраженная обратная зависимость между величиной локального К-индекса и вероятностью регистрации РСС на частотах выше 10 Гц: чем выше возмущенность магнитного поля, тем меньше вероятность регистрации. Такая зависимость верна также и для общей вероятности регистрации РСС (вне зависимости от частотного диапазона).

\section{3. Методика оценки критической частоты слоя $\mathrm{F} 2$ по данным регистрации ИАР}

Как говорилось выше, одним из основных параметров, определяемых при мониторинге ИАР, является разница частот между соседними резонансным максимумами - $d F$. Для оценки этой величины, согласно (Поляков и др., 1981), можно использовать следующее выражение:

$$
d F=\frac{c}{2 n_{A} L},
$$

где: с - скорость света; $L=h+l$ - линейный размер резонатора, обусловленный толщиной главного ионосферного максимума $-h$ и характерным масштабом спада альфвеновского 
А.В. КоЛосков: ОПРЕДЕЛЕНИЕ КРИТИЧЕСКОЙ ЧАСТОТЫ Ғ-СЛОЯ ПО ДАННЫМ ...

показателя преломления выше максимума $\mathrm{F} 2$-слоя $-l ;{ }_{n_{A}}-$ альфвеновский показатель преломления в максимуме слоя F2, который рассчитывается как:

$$
n_{A}=\frac{c \sqrt{4 \pi \rho}}{B_{0}} \propto \sqrt{M_{e f f} \cdot N_{e \max }},
$$

где: $B_{0}$ - напряженность магнитного поля Земли; $\rho$ и $N_{e \max }-$ плотность плазмы и электронная концентрация на высоте максимума слоя F2; $M_{e f f}$ - эффективная масса ионов; $f_{0} F 2$ - критическая частота в максимуме слоя $\mathrm{F} 2$. В свою очередь известно, что электронная концентрация является квадратичной функцией от плазменной частоты $f_{p}$ (Jayachandran et al., 2004):

$$
N_{e}=0.0124 \cdot f_{p}^{2} .
$$

Поэтому для электронной концентрации в максимуме слоя F2 - $N_{\text {emax }}$ можно написать следующее уравнение: $N_{e \max }=0.0124 f_{0} F 2^{2}$. Таким образом, учитывая (2-3) и предполагая, что ионосферная плазма является однокомпонентной, получаем:

$$
n_{A} \propto f_{0} F 2 .
$$

С другой стороны отметим, что ионосферный слой можно характеризовать не только критической частотой $f_{0} F 2$, но и величиной ПЭС. Двумя этими параметрами определяется толщина ионосферы $L$. Для оценки данной величины будем следовать модели прямоугольной ионосферы, которую можно найти в работе (Jayachandran et al., 2004). Будем считать, что электронная концентрация в ионосферном слое толщиной $L$ является константой, равной электронной концентрации в максимуме $N_{\text {emax }}$ (т.е. ионосфера является однородной). При этом полное электронное содержание такой модельной ионосферы равно полному электронному содержанию реальной ионосферы. В таком модельном приближении толщина ионосферы $L$ совпадает с линейным размером альфвеновского резонатора и, согласно работе (Јауасhandran et al., 2004), рассчитывается как:

$$
L=\alpha \frac{\Pi \ni C}{f_{0} F 2^{2}}, \text { где } \alpha \text { - масштабный коэффициент. }
$$

Согласно (5), ПЭС пропорционален электронной концентрации в максимуме F2-слоя и, соответственно, связан с критической частотой слоя F2 квадратичной зависимостью:

$$
\Pi Э С \approx N_{e \max } \approx f 0 F 2^{2} .
$$

Анализ выражений (1-6) позволяет установить качественную связь между разницей частот резонансных мод ИАР - $d F$, критической частотой слоя $\mathrm{F} 2$ - $f_{0} F 2$ и полным электронным содержанием - ПЭС:

$$
d F \propto 1 / f 0 F 2 \propto 1 / \sqrt{\Pi Э C} .
$$

Ниже приведены экспериментальные данные, подтверждающие правомочность использования такой простой физической модели. На рис. 3 изображены определенные экспериментально зависимости для среднесуточных величин $d F$ и $f_{0} F 2$ в Антарктике за весь рассматриваемый период и в среднем за сутки. На обоих графиках хорошо прослеживается антикорреляция двух кривых.

На рис. 4 показана оценка зависимости между $d F$ и $f_{0} F 2$ (сплошная кривая), рассчитанная по данным экспериментальных наблюдений на УАС.

Исходя из зависимостей (1-7) и экспериментальных данных, которые получены на НЧО (рис. 3-4), авторами работы предложена простая феноменологическая физическая модель связи критической частоты ионосферы $f_{0} F 2$ и разности частот между резонансными модами ИАР - $d F$ : 
$d F=-\alpha \cdot f_{0} F 2+\beta$.

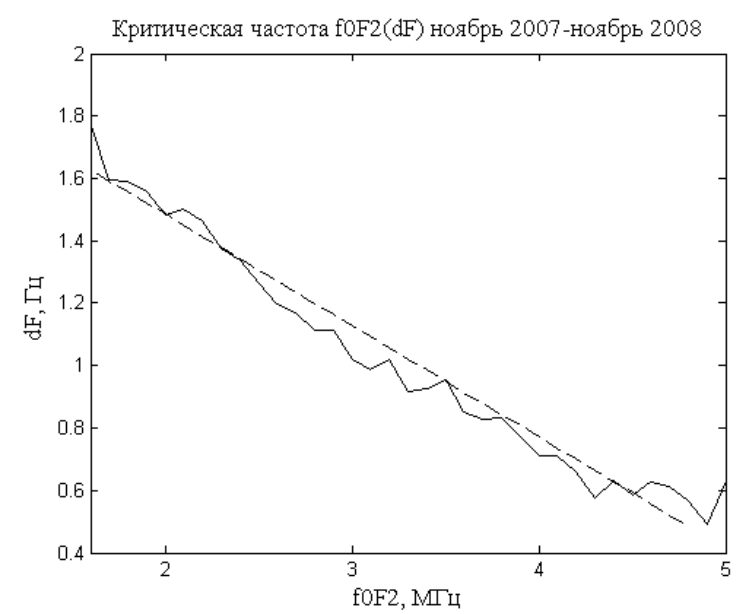

Рис. 4. Экспериментальная зависимость между $\mathrm{dF}$ и $f_{0} F 2$, вычисленная в соответствии с данными наблюдений на УАС с 1 ноября 2007 г. по 30 сентября 2008 г. (сплошная кривая), и ее модельное линейное представление (штриховая линия).

Для расчета коэффициентов, входящих в (8), в экспериментальную зависимость $f_{0} F 2=f(d F)$ (сплошная кривая на рис. 4) методом минимума квадратов отклонений была вписана линейная функция (пунктирная линия). Согласно полученной модельной формуле была восстановлена критическая частота слоя F2 по данным наблюдений ИАР и проведено сравнение со значениями $f_{0} F 2$, которые получены на УАС с помощью ионозонда. Результат сравнения представлен на рис. 5: слева - за весь период наблюдений, справа увеличенный фрагмент участка в 8 суток (с 15 по 22 ноября 2008 г.). Синим цветом показана критическая частота по данным ионозонда, красным - восстановленная по $d F$. Из рисунка видна высокая степень подобия восстановленных и измеренных величин $f_{0} F 2$. Коэффициент корреляции между ними составил 0.8 .

\section{4. Выводы}

Мониторинг ИАР, организованный на высокоширотной обсерватории УАС и среднеширотной НЧО, является эффективным средством диагностики геокосмоса и изучения свойств естественных УНЧ шумов в полярном и среднеширотном регионах. В работе проведён анализ морфологических особенностей характеристик резонанса на длительном массиве данных, подтверждены полученные ранее другими авторами сезонные и суточные зависимости параметров РСС. Уточнён частотный диапазон существования альфвеновского резонанса - впервые обнаружены РСС выше 10 Гц, проанализированы условия возникновения подобного эффекта. Предложена и обоснована новая методика оценки F0F2 по данным наблюдений собственных частот ИАР. Достоверность методики подтверждена на значительном объеме экспериментальных данных, полученных для различных ионосферных условий и сезонов года.

\section{Благодарности}

Авторы выражают благодарность специалистам НАНЦ, ЛЦ ИКИ и РИНАНУ за помощь при монтаже и обслуживании приемных установок в Антарктике и в 
Украине. Работа выполнена при частичной финансовой поддержке НАНЦ в рамках НТР "Гелеомакс" - 0111U007683, а также ведомственных конкурсных НИР НАН Украины "Ятаган-2" 0111U000063, "МАИС" 01110004496 и "Шпицберген" 0111 U003977.

\section{Список литературы}

П.П.Беляев, С.В.Поляков, В.О.Рапопорт и др. Экспериментальные исследования резонансной структуры спектра атмосферного электромагнитного шумового фона в диапазоне короткопериодных геомагнитных пульсаций. Известия высших учебных заведений, 1989, т. 32, № 6, стр. 663-672.

П.П.Беляев, С.В.Поляков, В.О. Рапопорт и др. Теория формирования резонансной структуты спектра атмосферного электромагнитного шумового фона в диапазоне короткопериодных геомагнитных пульсаций. Известия высших учебных заведений, 1989, т. 32, № 7, стр. 802-810.

А.В. Колосков, В.Г. Синицын, Н.Н. Герасимова, Ю.М. Ямпольский. Околоземные резонаторы СНЧ-волн как индикаторы космической погоды. Космічна наука і технологія, 2008. Т. 14, № 5, стр. 49-64.

А.А. Остапенко, С.В. Поляков. Динамика коэффициента отражения альфвеновских волн диапазона Pc1 от ионосферы при вариациях электронной концентрации нижней ионосферы. Геомагнетизм и Аэрономия, т. 30, № 1, 1990, стр. 50-56.

С.В. Поляков, В.О. Рапопорт. Ионосферный Альфвеновский Резонатор. Геомагнетизм и Аэрономия, 1981, т. 21, стр. 816-822.

В.Г. Синицын, Ю.М. Ямпольский. УНЧ-СНЧ-резонансы в геокосмосе как индикаторы космической погоды. Лекции БШФФ-2006. С. 54-57.

P.P. Belyaev, T. Bosinger, S.V. Isaev et al., First evidence at high latitudes for the ionospheric Alfven resonator. Journal of geophysical research, vol. 104, \#A3, pp. 4305-4317, 1999.

P.P. Belyaev, S.V. Polyakov, E.N. Ermakova et al., Solar cycle variations in the ionospheric Alfvén resonator 1985-1995. Journal of Atmospheric and Solar-Terrestrial Physics 62, 2000, pp. 239248

T. Bosinger, C. Haldoupis, P.P. Belyaev et al., Spectral properties of the ionospheric Alfvén resonator observed at a low-latitude station $(\mathrm{L}=1.3)$. Journal of geophysical research, vol. 107, \#A10, 1281, 2002.

B. Jayachandran, T.N. Krishnankutty, and T.L. Gulyaeva, Climatology of ionospheric slab thickness. Annales Geophysicae, \#22, pp. 25-33, 2004.

S.R. Hebden, T.R. Robinson, D.M. Wright et al., A quantitative analysis of the diurnal evolution of Ionospheric Alfven resonator magnetic resonance features and calculation of changing IAR parameters. Annales Geophysicae, \#23, pp. 1711-1721, 2005.

A. Odzimek, A. Kulak, A. Michalec et al., An automatic method to determine the frequency scale of the ionospheric Alfven resonator using data from Hylaty station, Poland. Annales Geophysicae, \#24, pp. 2151-2158, 2006.

A. Parent, I.R. Mann, and K. Shiokawa, Observing the MLT and L-shell dependence of ground magnetic signatures of the ionospheric Alfven resonator. Int. Conf. Substorms-8, pp. 225-230, 2006.

O.A. Pokhotelov, F.Z. Feygin, Yu.G. Khabazin et al., Observations of IAR spectral resonance at a large triangle of geophysical observatories. Physics of Auroral Phenomena, Proc. XXVI Annual Seminar, Apatity, pp. 123-126, 2003.

A.G. Yahnin, N.V. Semenova, A.A. Ostapenko et al., Morphology of the spectral resonance structure of the electromagnetic background noise in the range of $0.1-4 \mathrm{~Hz}$ at $\mathrm{L}=5.2$. Annales Geophysicae \#21, pp. 779-786, 2003. 
А.В. Колосков, Н.А. Бару

ОПРЕДЕЛЕНИЕ КРИТИЧЕСКОЙ ЧАСТОТЫ Ғ-СЛОЯ ПО ДАННЫМ

НАБЛЮДЕНИЙ ИОНОСФЕРНОГО АЛЬФВЕНОВСКОГО РЕЗОНАНСА

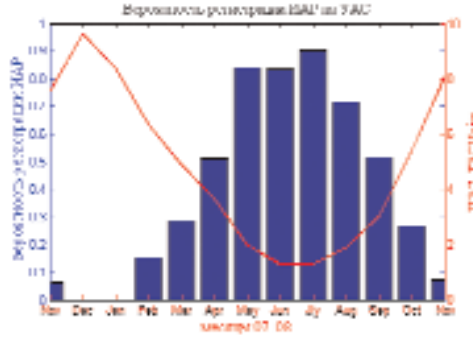

a)

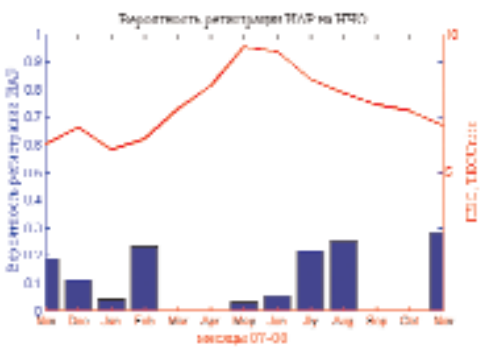

б)

Рис. 1. Среднемесячная вероятность регистрации ИАР за годовой интервал с ноября 2007 по ноябрь 2008: а) в Антарктиде, б) в Украине

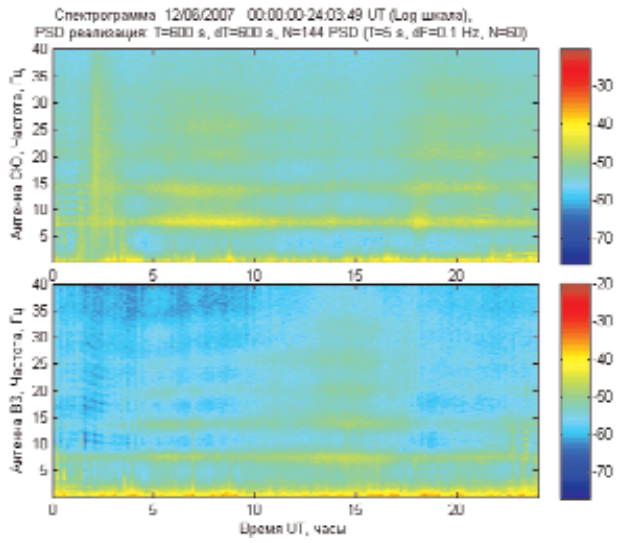

Рис. 2. Спектрограмма, полученная на НЧО для 6.12.2007, когда РСС наблюдалась на рекордно высоких частотах (0-3 UT канал восток-запад)

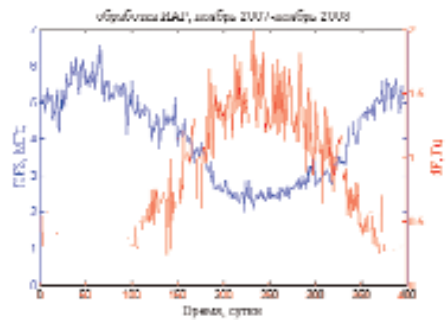

a)

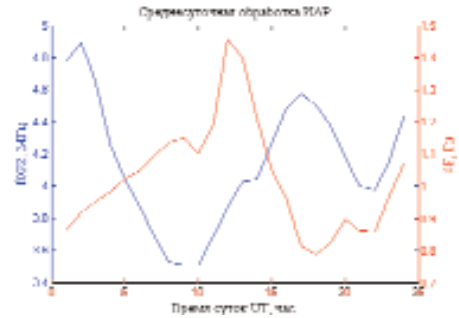

б)

Рис. 3. Полученные на УАС зависимости для величин $d F$ (красные кривые) и $f_{0} F 2$ (синие кривые): а) за период с 1 ноября 2007 г. по 30 сентября 2008 г., б) среднесуточный график за этот же промежуток времени

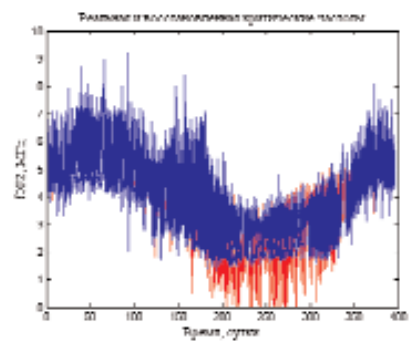

a)

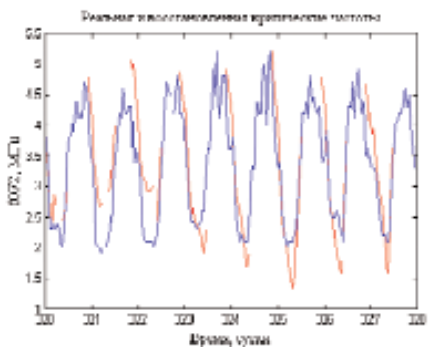

б)

Рис. 5 а) Критическая частота слоя F2 за период с 1 ноября 2007 г. по 30 сентября 2008 г., измеренная ионозондом (синим цветом) и воссозданная по данным наблюдений ИАР (красным); б) увеличенный фрагмент части рисунка с 15 по 22 ноября 2008 г. 